\title{
Pointlike Electric Charge in Gravitational Field Theory
}

\author{
H. Dekker
}

Delta Institute for Theoretical Physics, University of Amsterdam, Science Park, The Netherlands

Private Institute for Advanced Study, Résidence Le Jardin, Amsterdam, The Netherlands

hadekk944@gmail.com

\begin{abstract}
The existence of charged elementary 'point particles' still is a basically unsolved puzzle in theoretical physics. The present work takes a fresh look at the problem by including gravity-without resorting to string theory. Using Einstein's equations for the gravitational fields in a general static isotropic metric with the full energymomentum tensor (for the charged material mass and the electromagnetic fields) as the source term, a novel exact solution with a well-defined characteristic radius emerges where mass and charge accumulate: $r_{c}=\sqrt{T_{e} r_{0} / 2}$ - with $r_{e}=Q^{2} / 4 \pi \varepsilon_{0} m c^{2}$ being the 'classical' radius associated with the total charge $Q$ and where $r_{0}=2 m G / c^{2}$ is the Schwarzschild radius belonging to the observable mass $m$ (for the electron one has $r_{\mathrm{e}} \approx 10^{-15} \mathrm{~m}$ and $r_{0} \approx 10^{-57} \mathrm{~m}$ ). The resulting 'Einstein-Maxwell' gravitational electron radius can also be written as $F_{\mathrm{c}}=l_{\mathrm{P}} \sqrt{\alpha_{\mathrm{e}}}$, where $\ell_{\mathrm{p}}=\sqrt{h G / c^{3}} \approx 10^{-35} \mathrm{~m}$ is the fundamental Planck length and $\alpha_{\mathrm{e}}=e^{2} / 4 \pi \varepsilon_{0} \hbar c \approx$ $1 / 137$ the fine-structure constant, which yields $r_{\mathrm{c}}^{\text {electron }}=1.38063 \times 10^{-36} \mathrm{~m}$.
\end{abstract}

\section{Keywords:}

Point charge; self-gravitation; novel exact solution; Einstein-Maxwell electron radius; renormalization

PACS numbers: $04.20 . \mathrm{Cv}, 04.20 . J b, 04.20 .-\mathrm{q}, 04.40 . \mathrm{Nr}, 04.40 .-\mathrm{b}$

Date of Submission: 30 August, 2018

DOI: 10.24297/jap.v14i2.7596

ISSN: $2347-3487$

Volume: 14 Issue: 2

Journal: Journal of Advances in Physics

Website: https://cirworld.com

This work is licensed under a Creative Commons Attribution 4.0 International License. 


\section{Introduction}

Modern theoretical physics is essentially based on the existence of a finite set of elementary 'point particles'-leptons and quarks-and their electromagnetic, gravitational, and weak or strong interactions (see, e.g., Refs. [1-5]). Apart from the neutrino's, all fundamental particles carry an electric charge. However, the very concept of a stable 'point charge'-such as the electron-is an old and as yet basically unsolved problem. Namely, why should it be possible to accumulate a finite amount of electric charge in an infinitely small volume? What internal force does the work against the repulsive self-interaction? In fact, without such a force the charged particle should immediately explode.

Historically, the problems with a point charge were already recognized in classical physics (see, e.g., Refs. [6-12]). For instance, in Poincaré's 'electron model' [7] the electric force on the charged sphere was counteracted by an elastic force of unspecified, non-electromagnetic and non-gravitational nature in order to define a total energy-momentum tensor $T_{\ltimes \beta}$ satisfying the condition $\partial T_{\propto \beta} / \partial x_{\beta}=0$ characteristic of a closed system (see, e.g., Ref. [13], Ch. 7.3). In fact, even more than a decade after the advent of general relativity, during a visit to Leiden University in 1925, Einstein asked Lorentz' opinion on a purely electromagnetic model for the electron-i.e., without gravitational forces. Lorentz, however, rejected the idea (Ref. [12], Letter 398).

In any case, to quote from Feynman's Lectures (Ref. [10] Vol. I, p. 32-4): "The classical electron radius $F_{\mathrm{e}}=e^{2} / 4 \pi \varepsilon_{0} m c^{2} \approx 10^{-15} \mathrm{~m}$ no longer has the significance that we believe that the electron really has such a radius". More recently, based on state-of-the-art precision measurements of the electron's gyromagnetic $g$ factor (and using a simple 'electron model' due to Brodsky and Drell [14]), 1989 Nobel laureate H.G. Dehmelt has pointed out that "Today everybody 'knows' the electron is a Dirac point particle with radius $r_{8}=0$ and $g=2$. But is it? The value $r_{9} \approx 10^{-22} \mathrm{~m}$ given here constitutes an important new upper limit. [..] Thus, the electron may have size." (Ref. [15]; see also, e.g., Refs. [16, 17]).

Nevertheless, in non-gravitational quantum theory the electron can be treated successfully as a structureless point particle-at least, if the problem of its infinite self-energy is being 'swept under the carpet'. Indeed, as is well-known from the very beginning of quantum electrodynamics (see, e.g, Ref. [18]), this 'success' is only possible at a price. Namely, handling the infinite self-energy of a point charge requires an infinite mass renormalization to yield results in terms of the observed mass $m$ (see, e.g., Refs. [3,4,18-23]). Unfortunately, this fundamentally hampers the unification with quantum gravity - as the latter has been found to be non-renormalizable.

For instance, to quote from Ref. [3], p. 568: "The definition of quantum gravity beyond the formal level leads to a number of unsolved problems. [...] Even pure quantum gravity is non-renormalizable in four dimensions. In fact, it is commonly believed that the theory remains non-renormalizable, a property which would indicate the breakdown of local quantum theory at Planck's length scale $\ell_{\mathrm{p}}=\sqrt{h G / c^{2}} \approx 10^{-35} \mathrm{~m}$." It is further worth noticing that modern string theory has been proposed-and is still under development-inter alia to cope with the problem of point-like particles, replacing them by tiny one-dimensional loops of Planck size (see, e.g., Refs. $[4,5])$.

The present work takes a fresh look at the problem by including gravity-as yet without resorting to string theory, ignoring the weak interaction and without a priori assuming the mass-charge density to be rigorously zero outside some $x_{\mathrm{m}}$ (as in the usual Reissner-Nordström analysis; see, e.g., Refs. $[13,24,25]$ and Appendix A). Namely, the enormous amount of electrostatic energy compressed into an infinitely small volume must-according to Einstein's general theory of relativity-give rise to huge local gravitational effects. Therefore, in this article the gravitational field equations for the Ricci tensor $R_{\mu v}$ are studied for a classical selfgravitating charged mass, i.e., with the full energy-momentum tensor $T^{\mu v}$ for the material mass and the electromagnetic fields as the source of gravitational energy-in a static isotropic metric (Sec. II and III). The analysis involves an 'electrostatic equilibrium' condition (Sec. IV), and rigorously yields a well-defined novel mass-charge distribution with (in fact, for all charged leptons) a characteristic size $\tau_{\mathrm{c}} \approx 10^{-36} \mathrm{~m}$ (Sec. $\mathrm{V}$ and VI). 


\section{The gravitational field equations}

Throughout the present article, it is chosen to keep both the speed of light $c$ and the gravitational constant $G$ explicitly in the formula-rather than using 'geometrized units' where $c=G=1$. The notation closely follows that of Weinberg's book [1]. The field equations of general relativity may then be written as

$$
R_{\mu v}=-\frac{8 \pi}{e^{4}} G_{\mu v x}
$$

with

$$
G_{\mu v}=G\left(T_{\mu v}-\frac{1}{2} g_{\mu v} T_{\lambda}^{\lambda}\right)
$$

where $R_{\mu v}$ is the Ricci tensor, $T_{\mu v}=g_{\mu \lambda} g_{v K} T^{\lambda k}$ the covariant energy-momentum tensor, $g_{\mu v}$ the metric tensor, and $T_{\lambda}^{\lambda}=g^{\mu v} T_{\mu v}$ (with $g^{\mu v} g_{v d}=\delta^{\mu}{ }_{\lambda}$ ). In mixed components (see, e.g., Sec. IV), Eq. (2.1) becomes $R^{\mu}{ }_{v}=-\left(8 \pi / c^{4}\right) G^{\mu}{ }_{v}$ with $R^{\mu}{ }_{v}=g^{\mu \lambda} R_{\lambda v}$ and $G^{\mu}{ }_{v}=G\left(T^{\mu}{ }_{v}-\frac{1}{2} \delta^{\mu}{ }_{v} T^{\lambda}{ }_{\lambda}\right)$. The covariant tensor $g_{\mu v}$ defines the Riemannian space-time geometry by means of the proper time $d \tau$, such that

$$
d \tau^{2}=-c^{-2} g_{\mu v} d x^{\mu} d x^{v}
$$

where, in this paper, time will be labelled by $\mu=0$.

For the problem of a charged mass, the energy-momentum tensor consists of two contributions, viz., for the-electrically charged-matter and the electromagnetic field itself. In the standard 'ideal fluid' form (without internal pressure), the energy-momentum tensor for the mass reads

$$
T_{\mathrm{m}}^{\mu V}=\rho_{\mathrm{m}} \frac{d x^{\mu}}{d \tau} \frac{d x^{p}}{d \tau}
$$

where $\rho_{\mathrm{m}}=-c^{-2} T_{\mathrm{m} \lambda}^{\lambda}$ is the proper mass density. In the Dirac representation one has

$$
\rho_{\mathrm{m}}=g^{-1 / 2} \sum_{n} m_{n} \int \delta^{4}\left[x-x_{n}(\tau)\right] d \tau,
$$

where $g=\left\|-g_{\mu v}\right\|$ is the determinant of the metric tensor, so that the mass $M=\Sigma_{n} m_{n}$ is given by

$$
M=\int \gamma^{1 / 2} \rho_{\mathrm{mn}} d^{\mathrm{a}} x
$$

where $\gamma=-g / g_{00}$ is the determinant of the three-dimensional metric tensor $\gamma_{i j}=\left(g_{i j}-g_{0 i} g_{0 j} / g_{00}\right)$-see, e.g., Refs. $[13,26]$.

The system is closed (so that $T_{\text {total }_{y} v}^{\mu v}=0$ ) by including the electromagnetic field energy-momentum, given by 


$$
T_{\mathrm{em}}^{\mu v}=\varepsilon_{0}\left(F_{\lambda}^{\mu} F^{v \lambda}-\frac{1}{4} g^{\mu v} F_{\lambda K} F^{\lambda K}\right)
$$

where the antisymmetric electromagnetic field tensor $F^{\mu v}$ is defined by $F^{\text {oi }}=E_{\mathrm{i}}$ for the electric field and $F^{\mathrm{ij} j} / c$ $=\varepsilon_{i j k} B_{k}$ for the magnetic field, $\varepsilon_{i j k}$ representing the usual three-dimensional Levi-Civita symbol and $\varepsilon_{0}$ being the vacuum permittivity. It is useful to note that, since $g_{\mu v} F^{\mu}=F_{v a}$ and $g_{\mu v} g^{\mu v}=4$, Eq. (2.7) implies that $T_{\mathrm{em} \lambda}^{d}=g_{\mu v} T_{\mathrm{em}}^{\mu v}=0$.

The electromagnetic fields satisfy Maxwell's equations

$$
\frac{\partial}{\partial x^{p}}\left(g^{1 / 2} F^{\mu v}\right)=g^{1 / 2} j_{e n}^{\mu} / \varepsilon_{0}
$$

where $l_{\mathrm{em}}^{\mu}=c^{-1} \rho_{\mathrm{e}} d x^{\mu} / d \tau$ is the current four-vector-with $\rho_{\mathrm{e}}$ being the proper charge density, which in the Dirac representation reads

$$
\rho_{e}=g^{-1 / 2} \Sigma_{n} e_{n} \int \delta^{4}\left[x-x_{n}(\tau)\right] d \tau x
$$

Since the current satisfies the conservation law $l_{\mathrm{em} ; \mu}^{\mu}=0$, the charge $Q=\Sigma_{n} e_{n}$ is conserved and given by

$$
Q=\int \gamma^{1 / 2} \rho_{e} d^{9} x
$$

Note that both $\rho_{\mathrm{e}}$ and $\rho_{\mathrm{m}}$ transform like a scalar.

\section{The static isotropic case}

The static isotropic metric may be written in the 'standard' form in spherical coordinates $r, \theta, \varphi$, so that the only nonvanishing components of the metric tensor are

$$
g_{00}=-B(r), g_{r y}=A(r), g_{\theta \theta}=r^{2}, g_{\varphi \varphi}=r^{2} \sin ^{2} \theta_{x}
$$

and $g=A(r) B(r) r^{4} \sin ^{2} \theta$. The only nonzero components of the Ricci tensor are (with $A^{0}=d A / d r$, etc.)

$$
\begin{aligned}
& R_{00}=-\frac{B^{-}}{2 A}+\frac{B}{4 A}\left(\frac{A}{A}+\frac{B}{B}\right)-\frac{B^{-}}{r A^{x}} \\
& R_{r Y}=\frac{B^{-}}{2 B}-\frac{B^{-}}{4 B}\left(\frac{A}{A}+\frac{B^{-}}{B}\right)-\frac{A}{r A}
\end{aligned}
$$




$$
R_{\theta \theta}=-1-\frac{Y}{2 A}\left(\frac{A}{A}-\frac{B}{B}\right)+\frac{1}{A^{x}}
$$

while $R_{\varphi \varphi}=R_{\theta \theta} \sin ^{2} \theta$ and the only nonzero component of the material energy-momentum tensor now reads

$$
T_{\mathrm{m}}^{\infty 0}=\rho_{\mathrm{m}} c^{2} / B_{x}
$$

which readily yields

$$
\begin{gathered}
G_{00}^{\mathrm{m}}=\frac{1}{2} G B \rho_{\mathrm{m}} c^{2}, \quad G_{r \%}^{\mathrm{m}}=\frac{1}{2} G A \rho_{\mathrm{m}} c^{2}{ }_{x} \\
G_{\theta \theta}^{\mathrm{m}}=\frac{1}{2} G r^{2} \rho_{\mathrm{m}} c^{2},
\end{gathered}
$$

while $G_{\phi \varphi}^{\mathrm{m}}=G_{\theta \theta}^{\mathrm{m}} \sin ^{2} \theta$.

For the metric (3.1), Eq. (2.8) leads to the Poisson equation

$$
\frac{d}{d y}\left(r^{2} \sqrt{A B} E_{y}\right)=r^{2} \sqrt{A} \frac{p_{\text {ix }}}{\varepsilon_{\varphi}}
$$

for the only nonzero component $E_{y}=F^{\text {or }}$ of the electric field, while for the contributions from Eq. (2.7) one obtains

$$
\begin{gathered}
G_{00}^{\mathrm{em}}=\frac{\varepsilon_{0}}{2} G A B^{2} E_{\gamma}^{2}, \quad G_{r r}^{\mathrm{em}}=-\frac{\varepsilon_{0}}{2} G A^{2} B E_{\gamma}^{2}, \\
G_{\theta \theta}^{\mathrm{em}}=\frac{\varepsilon_{g}}{2} G A B r^{2} E_{\gamma x}^{2}
\end{gathered}
$$

while $G_{\varphi \varphi}^{\mathrm{em}}=G_{\theta \theta}^{\mathrm{em}} \sin ^{2} \theta$. Note that $G_{e m \lambda}^{\lambda l}=g^{\mu v} G_{\mu v}^{\mathrm{em}}=0$, as it should be.

\section{The equilibrium condition}

Einstein's gravitational field equations (2.1) for the static isotropic mass-charge system may thus be written as

$$
\frac{R_{m g}}{B}=-\frac{4 \pi}{c^{2}} G_{P_{\mathrm{m}}}-\frac{4 \pi \varepsilon_{g}}{e^{4}} G A B E_{y}^{2}
$$




$$
\begin{aligned}
& \frac{R_{\gamma T}}{A}=-\frac{4 \pi}{c^{2}} G \rho_{\mathrm{m}}+\frac{4 \pi \varepsilon_{g}}{c^{4}} G A B E_{\sigma x}^{2} \\
& \frac{R_{g Q}}{r^{2}}=-\frac{4 \pi}{c^{2}} G_{\rho_{\mathrm{m}}}-\frac{4 \pi \varepsilon_{g}}{c^{4}} G A B E_{\sigma x}^{2}
\end{aligned}
$$

Note that $R_{00} / B=-R^{0}{ }_{0}, R_{r v} / A=R^{r}{ }_{r}$, and $R_{\theta \theta} / r^{2}=R^{\theta}{ }_{\theta}$.

To find the equilibrium equation, first consider $R_{r y} / A+R_{00} / B$. This leads to

$$
\frac{1}{A}\left(\frac{A}{A}+\frac{B}{B}\right)=\frac{8 \pi}{E^{2}} G r \rho_{\mathrm{m}} x
$$

Using the $R_{\theta \theta}$-equation (4.3) to eliminate $A^{\circ}$, one gets

$$
\frac{Y}{A} \frac{B^{\prime}}{B}+\frac{1}{A}=1-\frac{4 \pi \varepsilon_{0}}{e^{4}} G A B r^{2} E_{\gamma}^{2}
$$

Now differentiating Eq. (4.5) with respect to $r$ and using $R_{r r} / A-R_{00} / B$ to eliminate $B$ ', one obtains

$$
\begin{aligned}
\frac{r B^{2}}{2 A B}\left(\frac{A}{A}+\frac{B}{B}\right)= & \frac{9 \pi \varepsilon_{g}}{e^{4}} G A B r E_{r}^{2} \\
& +\frac{4 \pi \varepsilon_{g}}{e^{4}} G \frac{d}{d r}\left(A B r^{2} E_{r}^{2}\right) .
\end{aligned}
$$

Once more invoking Eq. (4.4), the result reads

$$
\frac{B^{0}}{B}=\frac{2 A B r E_{r}^{2}+d\left[A B y^{2} E_{T}^{2}\right) / d r}{P_{m} e^{2} r^{2} / \varepsilon_{w}}
$$

which—putting $E=r^{2} \sqrt{A B} E_{y}$ and noticing the identity $d\left(E^{2} / r^{2}\right) / d r=-2 E^{2} / r^{2}+2\left(E / r^{2}\right) d E / d r$-by invoking the Poisson equation (3.7) can be rewritten as

$$
\frac{B}{B}=\frac{2 A \sqrt{B} E_{\mathrm{r}} p_{\mathrm{E}}}{p_{\mathrm{m}} E^{2}}
$$


which represents the balancing of the repulsive electrostatic self-force and the attractive gravitational selfforce. It is the electrostatic counterpart of the usual 'hydrostatic equilibrium' condition for ideal fluids. In fact, by virtue of the Bianchi identities, Eq. (4.8) is a direct consequence of the conservation law $T_{\text {total }}^{\text {rv }}=0$. Namely, one gets $T_{\mathrm{m} ;}^{r v}=\Gamma_{00}^{\gamma} T_{\mathrm{m}}^{00}=\Gamma_{00}^{r} P_{\mathrm{m}} c^{2} / B$ with $\Gamma_{00}^{\gamma}=B^{0} / 2 A$, while by means of the Poisson equation (3.7) one obtains $T_{\mathrm{E} m_{j} V}^{r v}=\sqrt{B} E_{r} \rho_{\mathrm{e}}$.

For a structureless charged mass the intrinsic charge-to-mass ratio $e_{n} / m_{n}$ should be an $n$ independent constant, i.e., $e_{n}=k m_{n}$, which according to Eqs. (2.5) and (2.9) implies the equation of state $\rho_{\mathrm{e}}=k \rho_{\mathrm{m}}$ for the proper density. Without loss of generality, one may put $k=Q / M$ so that-by virtue of Eq. (2.10) for $Q$ - the as yet undetermined mass $M$ satisfies Eq. (2.6). Hence, the equilibrium equation (4.8) may be rewritten as

$$
A E_{y}=\frac{\mu}{2} \frac{B^{0}}{B^{3 / 2^{x}}}
$$

with $\mu=M c^{2} / Q$. Now using the Newtonian limit $B=1-2 m G / r c^{2}$ and the Poisson limit $A E_{\gamma}=Q / 4 \pi \varepsilon_{0} r^{2}$ for $r \rightarrow \infty$, one obtains

$$
m=\frac{Q^{2}}{4 \pi \varepsilon_{Q} G M^{x}}
$$

By Eq. (4.9) the problem of the charged mass is reduced to finding the temporal metric function $B(r)$.

\section{The temporal metric function}

Consider Eq. (4.5) for $A$, and using Eq. (4.9) for $E_{\gamma}$ write it as

$$
A=1+\beta+\mathbf{M} \beta^{2}
$$

where $\beta=r B^{\circ} / B$ and $\mathbf{M}=\pi \varepsilon_{0} G \mu^{2} / c^{4}$. Now again take $R_{r z} / A-R_{00} / B$, collect the $A$ terms and once more use Eq. (4.9) for $E_{\gamma}$. After a somewhat laborious but otherwise elementary calculation this leads to

$$
\frac{F B^{-}}{B}+\left(1-\frac{1}{2} \beta\right) \frac{B^{-}}{B}-\left(1+\frac{1}{2} \beta\right) \frac{A}{A}=2 \mathbf{M} \beta \frac{B^{-}}{B^{*}}
$$

Substituting $A$ and $A$ from Eq. (5.1), and combining similar terms-some of which add up to zero-Eq. (5.2) is found to factorize such that for $\mathbf{M}=\frac{1}{4}$ it becomes a trivial zero identity (see Appendix A and, e.g., Ref. [27]) while for $\mathbf{M} \neq \frac{1}{4}$ it either leads to the trivial solution $B^{\circ}=0$ or to a nontrivial metric function $B(r)$ satisfying

$$
\frac{1}{2} r B^{\circ}+\left(1+\frac{1}{2} \mathbf{M} \beta^{2}\right) B^{\circ}=0,
$$


which is akin to the prototype equation $d \beta / d t=\beta^{a}$ for the temporal evolution of so-called 'finite-time blowup' processes in, e.g., chemistry and hydrodynamic turbulence (see, e.g., Ref. [28], p. 353).

Noticing that $B \beta^{\circ}=r B^{\circ}+(1-\beta) B^{\circ}$, using Eq. (5.3) for $B^{\prime \prime}$ and defining the auxiliary variable $\zeta(r)=1 / \beta$, one thus rigorously obtains

$$
r \zeta \zeta^{\circ}=\mathbf{M}+\zeta+\zeta^{2}
$$

The exact solution of Eq. (5.4) is given by

$$
\frac{1}{2} \ln \left(\mathrm{M}+\zeta+\zeta^{2}\right)+\frac{1}{2 \sqrt{\mathrm{M}-\frac{1}{4}}} \operatorname{arccot} \frac{\zeta+\frac{1}{2}}{\sqrt{\mathrm{M}-\frac{1}{4}}}=\ln \left(\frac{Y}{9}\right),
$$

where the integration constant has been set equal to the Schwarzschild radius $x_{0}=2 \mathrm{mG} / \mathrm{c}^{2}$ belonging to the observed mass $\mathrm{m}$ in order to satisfy the Newtonian limit $\zeta \approx r / r_{0}$ for large values of $r$.

For $r \rightarrow \infty$, one has $\zeta \approx r / r_{0} \rightarrow \infty$ as well. On the other hand, $\zeta \rightarrow 0$ for $r \rightarrow r_{c}$. Namely, expanding Eq. (5.5) in powers of $\zeta$ yields $\zeta^{2} / 2 \mathbf{M} \approx \ln \left(r / r_{\mathrm{c}}\right)$, with

$$
r_{c}=r_{0} \sqrt{\mathrm{M}} \exp \left(\frac{1}{2 \sqrt{\mathrm{M}-\frac{1}{4}}} \operatorname{arccot} \frac{1}{2 \sqrt{\mathrm{M}-\frac{1}{4}}}\right) .
$$

Using $\mu=M c^{2} / e$ [as given below Eq. (4.9)] in the definition of $\mathbf{M}$ [as given below Eq. (5.1)] and invoking Eq. (4.10) for $M$, one obtains $\mathbf{M}=M / 4 m$. Once more using (4.10), this becomes $\mathbf{M}=Q^{2} / 16 \pi \varepsilon_{0} G m^{2}$ which leads to $\mathbf{M}=r_{\mathrm{e}} / 2 r_{0}$ - with $r_{\mathrm{e}}=Q^{2} / 4 \pi \varepsilon_{0} m c^{2}$ being the 'classical' radius belonging to the charge $Q$ and where $r_{0}=2 m G / c^{2}$ is the Schwarzschild radius belonging to the mass $m$-so that $r_{0} \approx \sqrt{T_{0} T_{\mathrm{E}} / 2}$, which explicitly amounts to

$$
r_{C}=|Q| \sqrt{\frac{G}{4 \pi \varepsilon_{0} e^{4}}} x
$$

where the exponential factor from Eq. (5.6) has been omitted as it is always of the order of unity. Actually, with $r_{\mathrm{e}} \approx 10^{-15} \mathrm{~m}$ and $r_{0} \approx 10^{-57} \mathrm{~m}$ for the electron, one has $\mathbf{M}=\tau_{\mathrm{e}} / 2 \tau_{0} \approx 10^{42}$ so that the exponential correction tends to $\exp (\pi / 4 \sqrt{\mathrm{M}}) \approx 1+O\left(10^{-21}\right)$. Finally, one obtains $r_{\mathrm{c}}^{\text {electron }} \approx 10^{-86} \mathrm{~m}$-which may be called the 'Einstein-Maxwell' gravitational electron radius.

\section{Results}

The temporal metric function now follows from $d B / d \zeta=B / r \zeta \zeta$. Using Eq. (5.4) for $\zeta^{\prime}$, this yields 


$$
B=\exp \left(-\frac{1}{\sqrt{M-\frac{1}{4}}} \operatorname{arccot} \frac{\zeta+\frac{1}{2}}{\sqrt{\mathrm{M}-\frac{1}{4}}}\right)
$$

where $\zeta\left(r / r_{\mathrm{c}}\right)$ follows from Eqs. (5.5) and (5.6), while $B\left(r \leq r_{\mathrm{c}}\right)=B_{\mathrm{c}}$. Notice that for finite values of $r / r_{\mathrm{c}}$ and $\mathbf{M} \rightarrow \infty$ one has $\zeta / \sqrt{\mathbf{M}}=\sqrt{\left(r / r_{c}\right)^{2}-1}$, so that the function $B(r)=\left(B-B_{c}\right) /\left(1-B_{c}\right)$ becomes $B=(2 / \pi) \arccos \left(r_{c} / r\right)$. Fig. 1 shows the exact solution $B(r)$ of Eq. (5.3) for a few values of M. Further, using Eq. (5.5) for $\zeta$, Eq. (6.1) can also be written as $B(r)=\left(r_{0} / r\right)^{2}\left(\mathbf{M}+\zeta+\zeta^{2}\right)$. Hence, $B_{\mathrm{c}}=\left(r_{0} \sqrt{\mathbf{M}} / r_{\mathrm{c}}\right)^{2}$ while a simple calculation using Eqs. (5.4) and (5.5) yields $B^{\prime}\left(r \rightarrow r_{\mathrm{c}}\right) \propto 1 / \sqrt{r-r_{\mathrm{c}}}$.

Similarly, the radial metric function $A(r)$ follows from Eq. (5.1) as

$$
\frac{1}{A}=\frac{\zeta^{2}}{\mathrm{M}+\zeta+\zeta^{2}}
$$

while $A\left(r<T_{\mathrm{c}}\right)=1$. For finite $r / T_{\mathrm{c}}$ and $\mathbf{M} \rightarrow \infty$, one gets $1 / A=1-\left(T_{\mathrm{c}} / r\right)^{2}$.

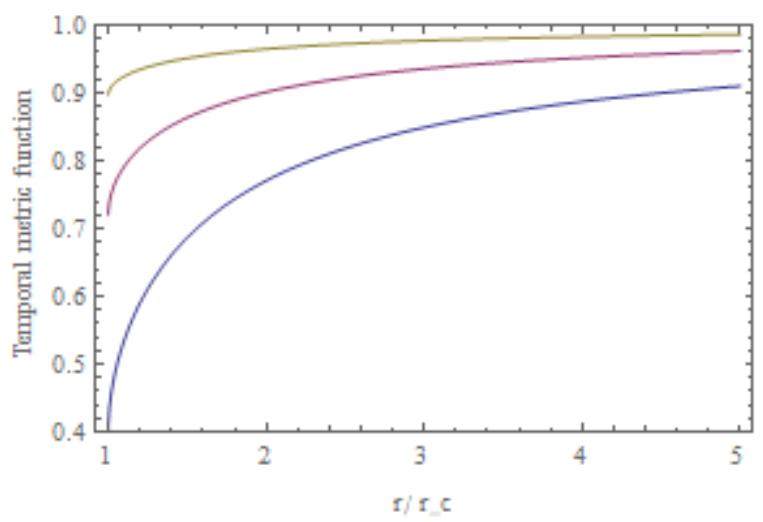

Figure 1. The temporal metric function $B(r)$ [for $\mathbf{M}=2$ (bottom line), $\mathbf{M}=20$ (middle), $\mathbf{M}=200$ (top)]. Shown is the numerical solution of the exact Eq. (5.3), as a function of the non-dimensional radial variable $r / T_{\mathrm{c}}$.

Next, the radial electrostatic field is obtained from Eq. (4.9), which yields

$$
E_{\gamma}=\frac{\mu}{2 r \sqrt{B}} \frac{\zeta}{\mathrm{M}+\zeta+\zeta^{2}} \theta\left(r-r_{\mathrm{c}}\right)
$$

where $\theta(r)$ is the unit step function. Fig. 2 shows the exact solution as $E_{F}(r) / E_{c}$, with $E_{c}=\mu / 2 r_{c} \sqrt{M}$. For finite values of $r / r_{\mathrm{c}}$ and with $\mathbf{M} \rightarrow \infty$, this function becomes $E_{r} / E_{\mathrm{c}}=\left(r_{\mathrm{c}} / r\right)^{2} \sqrt{1-\left(T_{\mathrm{c}} / r\right)^{2}}$, which has its peak value $\frac{2}{9} \sqrt{3} \approx 0.38$ at $r / r_{\mathrm{c}}=\frac{1}{2} \sqrt{6} \approx 1.22$. One further gets

$$
r^{2} \sqrt{A B} E_{y}=\frac{\mu}{2} \frac{r}{\sqrt{\mathrm{M}+\zeta+\zeta^{2}}} \Theta\left(r-r_{\mathrm{c}}\right)
$$


Finally, the Poisson equation (3.7) leads to

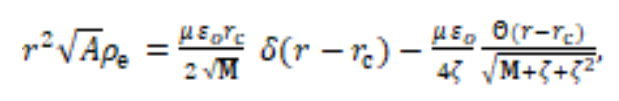

where $\delta(r)$ is the Dirac distribution and which rigorously satisfies Eq. (2.10) for the total charge $Q$-recalling that $\mu=M c^{2} / Q$ [see its definition below Eq. (4.9)] with $M c^{2}=Q^{2} / 2 \pi \varepsilon_{0} r_{0}$ [from Eq. (4.10) and $r_{0}=2 m G / c^{2}$ ] and using Eq. (5.6) for $r_{c}$, so that

$$
\frac{\mu \varepsilon_{0} Y_{c}}{2 \sqrt{\mathrm{M}}}=\frac{Q}{4 \pi} \exp \left(\frac{1}{2 \sqrt{\mathrm{M}-\frac{1}{4}}} \operatorname{arccot} \frac{1}{2 \sqrt{\mathrm{M}-\frac{1}{4}}}\right) .
$$

For finite values of $r / r_{\mathfrak{c}}$ and $\mathbf{M} \rightarrow \infty$, the second part of the density (6.5) now becomes $\rho_{\mathbb{e}}^{\text {cont }}=$ $-\left[Q / 8 \pi r_{c}^{3} \sqrt{\mathbf{M}}\right]\left(\mathrm{r}_{\mathrm{c}} / r\right)^{4}$. Clearly, with $\mathbf{M} \approx 10^{42}$ [see below Eq. (5.7)] charge and mass almost completely accumulate at the Einstein-Maxwell radius $r_{c}^{\text {electron }}$ - the tail in Eq. (6.5) containing only some $10^{-21} Q$.

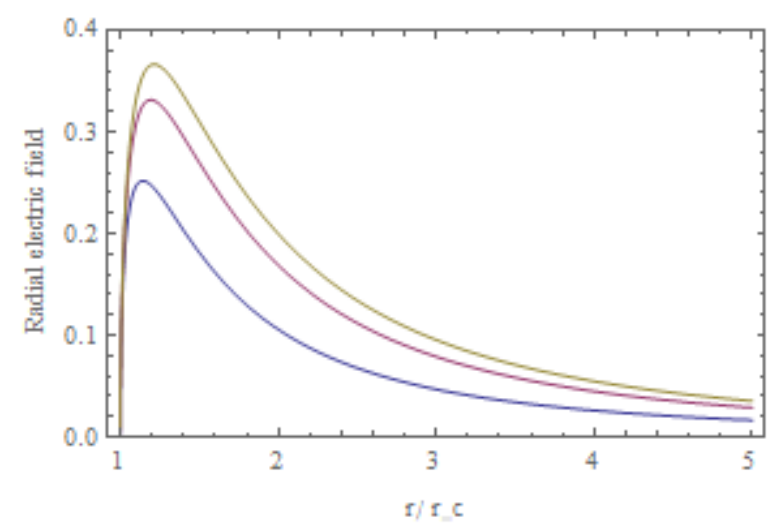

Figure 2. The radial electrostatic field $E_{\mathrm{r}}(r)$ [for $\mathbf{M}=2$ (bottom line), $\mathbf{M}=20$ (middle), $\mathbf{M}=200$ (top)]. Shown is $E_{\mathrm{r}} / E_{\mathrm{c}}$ [with $E_{\mathrm{c}}=\mu / 2 \mathrm{Mr}_{0}$ ], using the numerical solution of the exact Eq. (5.3), as a function of the nondimensional radial variable $r / r_{c}$.

The singular part of the density follows from the Poisson equation rather than from the gravitational field equations per se. Namely, from Eq. (6.5) one has $p_{e}^{\text {sing }}=\left(\mu \varepsilon_{0} / 2 M_{r_{c}}\right) \zeta(r) \delta\left(r-r_{c}\right)$, so that by virtue of $\zeta\left(r_{\mathrm{c}}\right)=0$ one has $T_{\mathrm{m}}^{\text {sing } 00}=0$. Of course, the continuous part of the density $\rho_{\mathrm{e}}^{\text {cont }}=-\mu \varepsilon_{0} / 4 r^{2} \zeta^{2} A$ obeys the Einstein equations for $r>r_{\mathrm{c}}$ (for all values of M). E.g., consider Eq. (4.4) and note that its right-hand side amounts to $-2 \mathbf{M} / r \zeta^{2} A$, so that it remains to show that $r\left(A^{0} / A+B^{0} / B\right)=-2 \mathbf{M} / \zeta^{2}$-which is easily done since $r A^{\prime} / A=\zeta d A / d \zeta=-1 / \zeta-2 \mathbf{M} / \zeta^{2}$ [by Eqs. (5.4) and (6.2)] and $r B^{\prime} / B=1 / \zeta$ by definition.

\section{Final remarks}

The mass-charge distribution (6.5) is an exact particle-like solution of the classical Einstein-Maxwell equations (for all values of the parameter $\mathbf{M}=Q^{2} / 16 \pi \varepsilon_{0} G m^{2}$ ). It emerges from a rigorous balance between electrostatic self-repulsion and gravitational self-attraction for all values of the radius $r$ (see Sec. IV), which is part of its novelty-see, e.g., Refs. [13, 24, 25]. It is further worth noticing that it appears to be the only solution for a point-like charge which correctly satisfies the observable Newtonian and Poisson limits for large 
$r$ but does not necessarily explode (as is, for instance, the case for the usual Reissner-Nordström 'superextremal' black hole electron; see, e.g., Ref. [24]).

For an elementary 'point charge'-like, e.g., the Dirac electron-the electrostatic self-interaction is the well-known source of an infinite self-energy (plaguing quantum field theory and its unification with general relativity), and it requires the inclusion of gravity to establish equilibrium at the new finite size $r_{c}=\sqrt{r_{\mathrm{e}} r_{0} / 2} \approx 10^{-36} \mathrm{~m}$ (where $r_{\mathrm{e}} \approx 10^{-15} \mathrm{~m}$ is the 'classical' electron radius and $r_{0} \approx 10^{-57} \mathrm{~m}$ is the electron Schwarzschild radius)—as shown at the end of Sec. V. In fact, a system of this kind was first considered by Poincaré as a model for the electron, however, without specifying the nature of the model's counteracting 'elastic forces' (see, e.g., Refs. [7, 13]).

In the present work it is shown that these forces intrinsically arise from local gravitational effects, which become huge when compressing a finite charge into an infinitesimal volume and which give rise to the novel 'hidden' mass $M=e^{2} / 4 \pi \varepsilon_{0} m G$ - which is essentially located at $r=r_{c}$ while taking care of the observable Newtonian and Poisson limits for $r \rightarrow \infty$. For the electron-with the parameter $\mathbf{M}=M / 4 m=r_{\mathbf{E}} / 2 r_{0} \approx 10^{42}$ (while, e.g., for the heavier tauon $\mathbf{M} \approx 10^{35}$ ) -mass and charge indeed almost completely accumulate at the radius $x_{c}$ (see Sec. VI).

By virtue of the relation (4.10) between the point-like massive charge $Q / M$ and the observable charged mass $m / Q$, the characteristic radius $r_{c}$ is mass independent (i.e., identical for all charged leptons). Namely, by Eqs. (5.6) and (5.7) one has $r_{c}=|e| \sqrt{G / 4 \pi \varepsilon_{0} c^{4}}$ - which is also worth noticing to follow at once from equating the electromagnetic mass $m_{\mathrm{c}}^{\text {charge }}=e^{2} / 8 \pi \varepsilon_{0} r_{\mathrm{c}} c^{2}$ at $r=r_{\mathrm{c}}$ to the Schwarzschild gravitational mass $m_{\mathrm{c}}^{\mathrm{grav}}=\tau_{\mathrm{c}} c^{2} / 2 G$ (so that for the parameter $\mathbf{M}$ one also has $1 / \sqrt{\mathrm{M}}=\mathrm{m} / \mathrm{m}_{\mathrm{c}}^{\mathrm{grav}}$, with $m_{\mathrm{c}}^{\mathrm{grav}} \approx 10^{-9} \mathrm{~kg}$ ). Hence, apart from involving the total charge, the novel 'Einstein-Maxwell' gravitational electron radius only depends on the fundamental constants $c$ and $G$. It is readily written as

$$
r_{\mathrm{c}}^{\mathrm{electron}}=\ell_{\mathrm{P}} \sqrt{\alpha_{\mathrm{e}}}
$$

where $\ell_{\mathrm{p}}=\sqrt{h G / c^{2}} \approx 10^{-95} \mathrm{~m}$ is the Planck length (see, e.g., Refs. $[3,11]$ ) and $\alpha_{\mathrm{e}}=e^{2} / 4 \pi \varepsilon_{0} h c \approx 1 / 137$ is the fine-structure constant. The resulting numerical value (see, e.g., Ref. [29]) is: $r_{c}^{\text {electron }}=1.38063 \times 10^{-36} \mathrm{~m}$.

Since the radius (7.1) is about an order of magnitude smaller than Planck's length $\ell_{\mathrm{p}}$-which is the fundamental size of the tiny one-dimensional loops proposed in modern string theory in order to inter alia cope with the problem of point-like particles (see, e.g., Refs. $[4,5]$ ) - the electron is indeed a point-like charge from the perspective of non-gravitational theory. However, while tiny it is large enough to produce only a relatively small quantum mechanical self-energy $\delta \mathrm{m}$.

For instance, for the free electron (see, e.g., Ref. [20], p. 270, tentatively taking $A \approx 1 / p_{\mathrm{c}}$ for the ultraviolet wave number cutoff) one now qualitatively gets $\delta m / m \approx\left(3 \alpha_{e} / 2 \pi\right) \ln \left(\ell_{\mathrm{c}} / r_{\mathrm{c}}\right) \approx 0.2$ (where $\ell_{\mathrm{C}}=\hbar / m c \approx 10^{-1 \mathrm{a}} \mathrm{m}$ is the Compton wavelength)—which removes the infinite mass renormalization from quantum electrodynamics (see also, e.g., Refs. $[22,23]$ ) and thus opens up new perspectives for unifying nongravitational quantum field theory with non-renormalizable quantum gravity.

\section{Appendix}

\section{The case $M=\frac{1}{4}$}

For $\mathbf{M}=\frac{1}{4}$ the upshot from Eq. (5.2) yields a trivial zero identity for all metric functions $B(r)$. Hence, in that case the problem can be solved by any mass-charge distribution, the ensuing metric following from the 
Poisson equation (3.7) with the equilibrium equation (4.9) for $E_{y}$ and Eq. (5.1) for $A(r)$. Upon once integrating the Poisson equation, one gets

$$
\frac{r}{r_{0}} \frac{\beta}{1+\frac{1}{3} \beta}=F_{e}(r)_{3}
$$

with $F_{e}=(4 \pi / Q) \int_{0}^{r} r^{2} \sqrt{A} \rho_{e} d r$-so that by Eq. (2.10) one has $F_{e}(\infty)=1$. For instance, for a density with finite range $\gamma_{\mathrm{m}}$ so that $\rho_{\mathrm{e}}\left(r>r_{\mathrm{m}}\right) \equiv 0$-such as, e.g., $4 \pi r^{2} \sqrt{A} \rho_{e}=Q \delta\left(r-r_{\mathrm{m}}\right)$-one would have $\beta=1 /\left(r / r_{0}-\frac{1}{2}\right)$ for $r>\gamma_{\mathrm{m}}$, and a metric singularity arises if $\gamma_{\mathrm{m}}<\frac{1}{2} \gamma_{0}$. With $\beta=r B^{v} / B$, this then leads to

$$
B=\left(1-\frac{1}{2} \frac{v_{g}}{r}\right)^{2}
$$

which is worth noticing to correspond to a special case (viz., the 'extremal black hole') of the ReissnerNordström metric [13, 24, 25].

A density with infinite range may be given by $F_{e}=r /\left(r+\varepsilon r_{0}\right)$-i.e., $4 \pi r^{2} \sqrt{A} \rho_{e}=\varepsilon r_{0} /\left(r+\varepsilon x_{0}\right)^{2}-$ which by Eq. (A.1) for $\mathbf{M}=\frac{1}{4}$ would imply $\beta=1 /\left[r / r_{0}+\left(\varepsilon-\frac{1}{2}\right)\right]$ and a singularity arises if $\varepsilon<\frac{1}{2}$. In this case the temporal metric function reads $B=\left[1+\left(\varepsilon-\frac{1}{2}\right) r_{0} / r\right]^{\left.-1 / t \varepsilon-\frac{1}{2}\right)}$, which for $\varepsilon \rightarrow 0$ of course reduces to the result given in Eq. (A.2).

Finally, notice that by Eq. (4.10) for $\mathbf{M}=\frac{1}{4}$ the observable classical mass amounts to $m=\mid Q \| \sqrt{4 \pi \varepsilon_{0} G}$, so that in this case the corresponding Schwarzschild radius $r_{0}=2 \mathrm{mG} / \mathrm{c}^{2}$ precisely equals the massindependent 'Einstein-Maxwell' radius $r_{\mathrm{c}}=|Q| \sqrt{G / 4 \pi \varepsilon_{0} c^{4}}=\ell_{\mathrm{p}} \sqrt{\alpha_{\mathrm{e}}}$ of Eq. (7.1). However, one now gets $m \approx 10^{-9} \mathrm{~kg}$ if $Q=e$-which is obviously far too heavy for the electron and is unlikely to be repaired by quantum effects (see, e.g., Sec. 7). To account for the correct order of magnitude of the electron mass $m \approx 10^{-30} \mathrm{~kg}$ one needs $\mathbf{M} \gg \mathbb{1}$, as shown in the main text.

\section{References}

[1] S. Weinberg, Gravitation and Cosmology (Wiley, New York, 1972).

[2] C.W. Misner, K.S. Thorne and J.A. Wheeler, Gravitation (Freeman, New York, 1970/2003).

[3] J. Zinn-Justin, Quantum Field Theory and Critical Phenomena (Oxford University Press, Oxford UK, 1989/2002) Fourth Edition.

[4] K.Becker, M. Becker and J.H. Schwarz, String Theory and M-Theory (Cambridge University Press, Cambridge UK, 2007).

[5] http://en.wikipedia.org/wiki/elementary_particle (2018) and /string_theory (2018).

[6] M. Abraham, Phys. Zeits. 5 (1904) 576.

[7] H. Poincaré, Rc. Circ. Mat. Palermo 21 (1906) 129.

[8] H.A. Lorentz, The Theory of Electrons (Teubner, Leipzig, 1909).

[9] G. Mie, Ann. Phys. 37 (1912) 511. 
[10] R.P. Feynman, Lectures on Physics (Addison-Wesley, Reading, 1963).

[11] A. Pais, Inward Bound (Clarendon Press, Oxford/New York, 1986).

[12] A.J. Kox, The Scientific Correspondence of H.A. Lorentz Vol. I (Springer, New York, 2008).

[13] C. Møller, The Theory of Relativity (Clarendon/Oxford University Press, Oxford/Delhi, 1952/1977).

[14] S.J. Brodsky and S.D. Drell, Phys. Rev. D 22 (1980) 2236.

[15] H.G. Dehmelt, in: High Energy Spin Physics, Edited by K. Heller (AIP Conf. Proc. No. 187, New York, 1989) p. 319; see also: NobelPrize.org/ Dehmelt-Lecture.pdf, 1989.

[16] B. Odom, D. Hanneke, D. D'Urso, and G. Gabrielse, Phys. Rev. Lett. 97030801 (2006).

[17] G. Gabrielse, D. Hanneke, T. Kinoshita, M. Nio, and B. Odom, Phys. Rev. Lett. 97030802 (2006).

[18] H.A. Kramers, Quantum Mechanics (Dover, New York, 1964) Ch. 89.

[19] N.G. van Kampen, Mat. Fys. Med. Kon. Dansk Vid.-Selsk. 26 (1951) 1.

[20] J.J. Sakurai, Advanced Quantum Mechanics (Addison-Wesley, Reading, 1967).

[21] L.D. Landau and E.M. Lifshitz, Quantum Electrodynamics, Course of Theoretical Physics Vol. 4 (Pergamon, Oxford, 1971/1994).

[22] H. Dekker, Physica A 133 (1985) 1, and quotes therein.

[23] H. Dekker, Intern. J. Mod. Phys. B 12 (1998) 965.

[24] H. Reissner, Ann. Phys. 50 (1916) 106, and http://en.wikipedia.org/wiki/reisner (2018).

[25] G. Nordström, Proc. Kon. Ned. Akad. Wetens., 20 (1918) 1238.

[26] L.D. Landau and E.M. Lifshitz, The Classical Theory of Fields, Course of Theoretical Physics Vol. 2 (Pergamon, Oxford, 1951/2003).

[27] R. Arnowitt, S. Deser and C.W. Misner, Phys. Rev. 120 (1960) 313.

[28] H. Dekker, in: Mathematical Modelling, edited by C.R. Brennan (Nova, New York, 2012), pp. 351-425.

[29] http://en.wikipedia.org/wiki/physical constants (2018). 